\title{
Lipid transfer proteins do their thing anchored at membrane contact sites... but what is their thing?
}

Louise $\mathrm{H}$ Wong $^{1}$ and Tim P Levine ${ }^{1 *}$

Address:

${ }^{1}$ Department of Cell Biology, UCL Institute of Ophthalmology, 11-43 Bath Street, EC1V 9EL, UK

*to whom correspondence should be addressed: tim.levine@ucl.ac.uk

ABSTRACT:

Membrane contact sites are dynamic structures where two organelles come close together to regulate flow of material and information between them. One type of inter-organelle communication is lipid exchange, which must occur for membrane maintenance and in response to environmental and cellular stimuli. Soluble lipid transfer proteins have been extensively studied, but additional families of transfer proteins have been identified that are anchored into membranes by transmembrane helices so that they cannot diffuse through the cytosol to deliver lipids. If such proteins target membrane contact sites they may be major players in lipid metabolism. The eukaryotic family of so-called Lipid transfer proteins Anchored at Membrane contact sites (LAMs) all contain both a sterol-specific lipid transfer domain in the StARkin superfamily (related to StART/Betv1), and one or more transmembrane helices anchoring them in the ER, making them interesting subjects for study in relation to sterol metabolism. They target a variety of membrane contact sites, including newly described contacts between organelles that were already known to make contact by other means. Lam1-4p target punctate ER-plasma membrane contacts. Lam5p and Lam6p target multiple contacts including a new category: Vacuolar non-NVJ cytoplasmic ER (VancE) contacts. These developments confirm previous observations on tubular lipid binding proteins (TULIPS) that established the importance of membrane anchored proteins for lipid traffic. However, the question remaining to be solved is the most difficult of all: are LAMs transporters, or alternately are they regulators that affect traffic more indirectly?

KEY WORDS:

Biological Transport

Ergosterol/analogs \& derivatives/chemistry

Intracellular Membranes/*metabolism

Membrane Lipids/metabolism

\author{
Saccharomyces cerevisiae \\ Proteins/genetics/metabolism \\ Vesicular Transport \\ Proteins/genetics $/{ }^{*}$ metabolism
}

\section{ABBREVIATIONS:}

Bin/Amphiphysin/RVS - BAR; endoplasmic reticulum - ER; ER-Mitochondria Encounter Structure -

ERMES; Glucosyltransferase, Rab-GTPase Activating protein and Myotubularin - GRAM; Lipid transfer protein Anchored at Membrane contact site - LAM; Lipid Transfer protein at Contact site - LTC; NucleusVacuole Junction - NVJ; Oxysterol Binding Protein - OSBP; Pleckstrin Homology - PH; Retinal

Degeneration B - RdgB; Steroidogenic Acute Regulatory lipid Transfer protein - StART;

StART/RHOalphaC/PITP/Bet v1/CoxG/CaIC - SRPBCC; Tubular Lipid binding protein - TULIP; Vacuolar and Mitochondrial Patch - vCLAMP; Vacuolar non-NVJ cytoplasmic ER - VancE. 
Intracellular lipid traffic is required in all cells that have two or more membrane-bound organelles. Since lipid modifying enzymes are predominantly targeted to a single compartment, the lipids must traffic to other membranes. Lipid traffic predates vesicle traffic, as it occurs in prokaryotes with two membranes. In eukaryotes some organelles are clearly not linked by vesicular traffic. Most obviously this applies to mitochondria, which must acquire most of their lipid precursors from the endoplasmic reticulum (ER) where most cellular lipids are synthesised, and this organelle pair was the first to be studied for intracellular lipid traffic ${ }^{[1]}$. Now many intracellular lipid transfer proteins have been identified, this designation having a strict in vitro definition (Box 1), and to date 15 distinct lipid transfer protein folds have been described (Table 1). The defining feature of these folds is an internal cavity, often capped with a flexible lid, the internal surfaces of cavity and lid being adapted to solubilise a water-insoluble molecule. A lipid transfer protein is therefore able to catalyse transfer through the cytosol, which otherwise presents a high energy barrier for lipids to move between membranes (Fig. 1A).

This review focusses on lipid transfer proteins that have transmembrane helices. As these proteins are anchored in membranes, they were overlooked in experiments on cytosolic lipid transfer proteins. Being integral membrane proteins, they are themselves unable to transfer from one organelle to another, hence according to the earliest dominant paradigm they would be unable to move lipids ${ }^{[2]}$. Anchored lipid transfer proteins could act to sense lipids to generate a signal, or present lipids to another protein, but transfer would not be feasible (Fig. 1B). The paradigm of soluble, cytoplasmic lipid transfer proteins was first called into question by the discovery that biosynthetic enzymes involved in ER-mitochondrial lipid exchange are concentrated at the membrane contact sites between these two compartments ${ }^{[3]}$. Membrane contact sites are found where two organelles come so close together that the gap (10-30 $\mathrm{nm}$ ) can be spanned by a single protein complex. They had been observed by early electron microscopy studies, but protein components were only discovered much later ${ }^{[4,5]}$. Many lipid transfer proteins have now been found at contacts: first RdgB (named because deletion in flies causes Retinal degeneration type $\underline{B}$ ) in flies ${ }^{[6]}$, then several oxysterol binding protein (OSBP) homologues in yeast ${ }^{[7]}$, and finally a large number of proteins with a shared motif to access the $E R^{[8]}$. As a result of this, the paradigm was revised to include lipid transfer by proteins pre-bound at membrane contact sites. Relatively static protein complexes might perform the entire lipid transfer reaction while simultaneously binding donor and acceptor membranes, reducing the time taken for diffusion 1000 -fold (Fig. 1C) ${ }^{[9]}$, which might be important for bulk sterol transport that is extremely fast ${ }^{[10]}$. Thus the prediction about lipid transfer proteins with TMHs changes from them being inactive to being hyperactive, as they cannot desorb from membranes into an inactive cytoplasmic pool. Among all lipid transfer proteins, transmembrane helices or hydrophobic hairpins are found in $>90 \%$ of tubular lipid binding proteins (TULIPs) ${ }^{[11]}$, a minority of Steroidogenic acute regulatory lipid transfer proteins (StARTs) ${ }^{[12]}$, and a few OSBP homologues ${ }^{[9]}$.

\section{Discovery of a new family of lipid transfer proteins}

In 2014 a new family of lipid transfer proteins was discovered in all eukaryotes using structural bioinformatics. The same approach had been applied previously to show that the synaptotagmin-like, mitochondrial, and lipid-binding protein (SMP) domain ${ }^{[13]}$ is a TULIP ${ }^{[14,15]}$. Next a search for domains in the plant protein VAD-1 (named for associated vascular associated death of leaves) identified a region structurally related to the helix grip domains in StARTs and Bet v1, and revealed a large eukaryotic family of proteins with the domain ${ }^{[16]}$. The helix grip domain is found in a large superfamily all with a seven stranded $\beta$ sheet wrapped around three $\alpha$ helices enclosing a large hydrophobic cavity. Different families within the superfamily are lipid transfer proteins, with ligands that include membrane lipids such as sterol. The structural homology of the new family to the helix grip superfamily was identified using i-TASSER, which predicts protein folds even when sequence homology is low ${ }^{[17]}$. Another group initiated searches with Lam6p, a previously unstudied yeast protein, to make the same identification ${ }^{[18]}$. Our group used HHsearch (which works similarly to i-TASSER ${ }^{[19]}$ ), to search in the opposite direction: searches initiated with StART and Bet v1 domains identified the new family ${ }^{[20]}$. Most eukaryotes have several proteins in the new family (Fig. 2), and budding yeast has twice the number (six) compared to closely related fungi (three) as a result of a recent genome duplication event in Saccharomyces ${ }^{[21]}$. The paralogous pairs are Lam1p/Sip3p, Ysp2p/Lam4p and Lam5p/Lam6p (Fig. 2). Lam1p was formerly called 
Ysp1p, but has been renamed for the sake of conformity within the family and ease of comprehension both inside and outside the field. Similarly, we suggest Ysp2p and Sip3p should be renamed Lam2p and Lam3 $p$, and we use those names hereafter. Although Lam1/3p show sequence divergence from other family members, Lam1/3p-like proteins are more prevalent in the NCBI database than proteins annotated as homologues of either Lam $2 / 4 p$ or Lam5/6p. These two pairs are more closely related to each other than to any other proteins in the family, indicating divergence from a single common fungal ancestor. The three human homologues, currently called GramD1a-c, are equally related to both Lam $2 / 4 p$ and Lam5/6p suggesting that they had a single common ancestor before the divergence of metazoans and fungi ${ }^{[20]}$. Although the cellular function of human LAMs has not yet been characterised, we anticipate all of them share properties with all of Lam2/4/5/6p. What little is known about the human homologues is that hLAMb (GramD1b) is strongly linked to solid and haematological tumours ${ }^{[22,}$ 23].

\section{Accompanying domains}

Proteins with the new helix grip domain are 600-1200 residues long, and the most distinctive feature is that $>99 \%$ have one or more transmembrane helix, a higher proportion than any other lipid transfer protein family. The transmembrane helices indicate permanent anchoring to a cell membrane, which is compatible with functions other than lipid transfer (Fig. 1B). The proteins also have other domains that are implicated in membrane targeting (Fig. 2). The most frequent occurrence is pleckstrin homology (PH)-like domain, related to those in Glucosyltransferases, Rab-GTPase Activating proteins and Myotubularins (GRAM). These domains are preceded by a short characteristic amphipathic helix (Fig. 2) that is also found in Rab-GTPase activating proteins and sterol glucosyltransferases, but not in myotubularins. Such domains are binding modules for various ligands, most of which are proteins rather than lipids ${ }^{[24]}$. The combination of $\mathrm{PH}$-like and helix grip domains is universal in eukaryotic evolution ${ }^{[16]}$. In addition there are other reversible membrane targeting domains, such as $\mathrm{PH}$ domains, Bin/amphiphysin/RVS (BAR) domains, C1 domains, C2 domains, SH3 domains and FYVE domains. Since the proteins are likely to be membrane embedded by their transmembrane helix, what is the need for other membrane targeting domains? They may well mediate targeting to a second membrane at contact site (Fig. 1C).

\section{Lipid specificity}

To verify the structural predictions, in vitro lipid binding and/or transfer assays were used to test for lipid interactions. Sterols were found as specific moderate affinity ( $\mathrm{Kd} \sim 1 \mu \mathrm{M})$ ligands for all proteins tested ${ }^{[18,20]}$. The domains appear to bind sterols (equally to cholesterol and ergosterol, the sterols in animals and yeast respectively) with no competition from phospholipids, although more detailed testing is needed ${ }^{[25,26]}$. Furthermore, the helix grip domains of any of Lam2/4/5/6p and of a human homologue rescued the phenotype in $L A M 1 / 2 / 3$ delete strains (see section on Function: precise positioning), indicating that all these domains may have the same specificity.

\section{Localisations}

Although the family is widespread in evolution, the only cell biological studies are in yeast ${ }^{[18,20,27]}$. With regards to their localisation, the take home message is that all six proteins are anchored in the ER, and all six target membrane contact sites. However, the message is complicated in that the six proteins come in two flavours. Lam1p, Lam2p, Lam3p and Lam4p all target contacts between the ER and the plasma membrane ${ }^{[20]}$. Expressed from their own promoters as GFP fusions, Lam2p is the most highly expressed, and the others are only just detectable by conventional confocal microscopy. The requirements for targeting have been studied extensively for Lam $2 p$ : the C-terminus alone produces cortical punctate targeting, which is reminiscent of the targeting by the viral protein TGBp3 of similar topology ${ }^{[28]}$. The predicted coiled-coil at the extreme C-terminus of Lam2/4p might also play a part in self-assembly (Fig. 2). To the extent that they have been studied, some of the proteins co-localise: Lam $2 p$ with Lam $4 p$, Lam $2 p$ with Lam3p, but the different expression levels indicate that they do not 
form 1:1 stoichiometric complexes. We speculate that all four proteins target the same super-complex, although no other components are known. One important feature of the contacts targeted by Lam1-4p has been determined: they are a unique type of ER-plasma membrane contact compared to others known previously, both in their highly punctate appearance (Fig. 3, image) and in their independence from known ER-plasma membrane tethers ${ }^{[29]}$.

Lam5p and Lam6p, the remaining two members of the family, target three other contact sites, but there is some doubt about the nature of the third contact. It is clear that they have two localisations. (i) ERmitochondrial contacts, with striking colocalisation of Lam6p and components of a complex found in many organisms, but not animals called the ER-mitochondria encounter structure (ERMES). This colocalisation dominates the picture when Lam6p is expressed at endogenous levels in cells growing in log phase ${ }^{[18,27]}$. (ii) The nucleus-vacuole junction (NVJ), where the outer nuclear envelope forms extended contacts with the yeast vacuole, which is equivalent to the mammalian lysosome ${ }^{[30]}$.

Another pool of contacts populated by Lam6p is of some interest. These are also on the vacuole, but these have been hard to identify with $100 \%$ certainty. Lam6p-Cherry was found in a similar distribution to Vps39p (also called Vam6p) ${ }^{[27]}$, which is the only known marker of vacuolar and mitochondrial patch (vCLAMP) contacts ${ }^{[31,32]}$. However, although Lam6p is close to Vps39p at the vacuole edge, the distributions of the two proteins are different. Also, under all other conditions Lam1-6p are entirely retained in the ER by their transmembrane helices, so it is unclear how they could be at VCLAMPs. To resolve this, we suggest the possibility that the peri-vacuolar patches of Lam6 $p$ all represent elements of the ER. The implication of this is that the ER contacts the vacuole at places apart from the NVJ. This new type of contact in yeast, for which we suggest the name Vacuolar non-NVJ cytoplasmic ER (VancE) contacts, not only contains Lam5/6p and Vac8p, but also the ER proteins Mdm1p and Nvj3p ${ }^{[33]}$. VancE contacts are prominent when GFP-Lam5/6p are over-expressed (Fig. 3 , image) ${ }^{[20]}$, but are also seen with endogenous level expression of GFP-Lam6 ${ }^{[18]}$. It has yet to be established if VancE contacts are indeed close to VCLAMPs, which would indicate the presence of zones of three-way contact between ER vacuole and mitochondria.

The new family of yeast proteins are all lipid transfer proteins at contact sites (Fig. 3), suggesting LTC as a generic acronym for the whole family ${ }^{[18]}$. Alternatively, the phrase lipid transfer proteins anchored at membrane contact sites (LAM) stresses membrane attachment as a functionally major feature. Since it was first conceived ${ }^{[34]}$, the superfamily of domains related to StART and Bet v1 domains has now acquired three new members: PRELI/Ups, LAMs and DUF3809 (Table 2), and we propose that the whole superfamily should be renamed as "StARkins" to reflect its increased breadth (Box 2).

\section{Interactions of LAM proteins}

No proteomic studies of Lam1-4p have yet been reported, but the ability of over-expressed Lam3p to redirect Lam2p away from cortical puncta, and Lam $2 p$-Lam $3 p$ colocalisation suggests that these two proteins may interact. For Lam6p much more is known. Proteomics approaches show that Lam6p interacts with the ERMES complex ${ }^{[18,27]}$. Importantly, the interaction with ERMES does not recruit Lam6p to ER-mitochondria contacts. Instead, extended proteomics studies showed that Lam6p binds to Tom70/71 $\mathrm{p}^{[18,27]}, 48 \%$ identical paralogues with the established function of recognising cargo for the TOM complex. The interaction was mapped to the PH-like domain of Lam6p, without which Lam6p targets exactly as if Tom70/71p are deleted: protein is re-routed to the NVJ and VancE contacts ${ }^{[18]}$. The proteomic study went even deeper, identifying the vacuolar binding partner of Lam6p (not via the PHlike domain) as Vac8p ${ }^{[18]}$, a peripheral membrane protein with many protein ligands already known, one of which is Nvj1p ${ }^{[30,35]}$. Therefore, Lam6p has receptors in two different organelles as well as being anchored into the ER, making it an ideal protein to integrate information about the status of multiple contact sites.

\section{Functional studies of LAM proteins:}




\section{Lam5/6p are tethers at multiple contact sites}

The relatively young field of membrane contact sites has focussed largely on what information or material might be exchanged there, with little attention paid to studying contact site regulation in response to changes in the environment and cell growth. Local regulation of contact sites might be detectable when one non-vesicular transfer route via one set of contacts compensates for the loss of another non-vesicular transfer route via another set of contacts. This was seen in the discovery of vCLAMPs as a pathway parallel to ERMES by which the secretory pathway communicates with mitochondria. In the absence of either complex the other enlarges, and loss of components of both contacts simultaneously is lethal ${ }^{[31,32]}$. New work on Lam6p shows that enlargement of either vCLAMP or ERMES contacts requires Lam6p, and over-expression of just this one protein when GFP-tagged (but interestingly not the untagged form) causes enlargement particularly of ER-mitochondria contacts, but also of NVJ, VCLAMP and VancE contacts, the latter two merging into a three way contact site $E R / m i t o c h o n d r i a / v a c u o l e{ }^{[27]}$. Thus, total expression and specific interactions of Lam6p regulate multiple contact sites, and this might be one way they are controlled during prolonged glucose starvation ${ }^{[36]}$.

The possibility that one or more of Lam1-4p has structural roles at ER-plasma membrane contacts has not been fully tested, but rapid over-expression of Lam2p makes no significant difference to the amount of cortical ER found close to the plasma membrane (Hayes and Levine, unpublished observations). This lack of obvious tethering ability is another way in which the ER-plasma membrane targeted proteins differ from Lam5/6p. It will be informative to pursue studies of both groups of LAM proteins further, because the human proteins appear to be equally related to both in terms of sequence, and may well combine functions and interactions of both types of yeast protein.

\section{Function in sterol metabolism}

All LAMs tested to date are sterol transfer proteins (in vitro, see Box 1), but that does not indicate their in vivo function. The most prominent route of sterol traffic in living cells is between the ER, where sterol is both made when the cell needs more and converted into neutral ester for storage outside membranes (in lipid droplets) when it is in excess ${ }^{[37]}$, and the plasma membrane, where sterol is enriched to 35\% total lipid ${ }^{[38]}$. Sterol traffic in both directions, forward (ER to plasma membrane) and reverse (plasma membrane to ER), has been assayed in strains with LAM deletions. For the forward assay, only a strain lacking LAM2 was tested, showing a non-significant minor reduction ${ }^{[20]}$. For the reverse assay, sterol traffic was reduced in strains lacking any one of $L A M 1, L A M 2$ or $L A M 3$ by $50 \%$ ( $\triangle$ lam 4 had no effect) ${ }^{[20]}$. The effect of deleting just one LAM is similar to inactivating all seven yeast OSBP homologues ${ }^{[39]}$.

Although these results show that the LAMs are important for sterol distribution in cells, multiple interpretations are possible, of which in vivo sterol traffic by LAMs is only one. If that were the case, maybe LAMs act in reverse traffic, but not in forward traffic. Only a few mechanisms for asymmetric transfer have been discovered ${ }^{[40]}$, but this is a plausible explanation. Linked to this, the physiological role of reverse traffic has not yet been fully resolved, in particular an explanation is needed for its very high capacity (in yeast $\geq 30,000$ sterols per cell per second, see Li and Prinz, 2004, Figure 1D ${ }^{[41]}$ ). A second variant of the trafficking interpretation is that the forward traffic assay somehow fails to reveal defects caused by loss of LAMs. No mutant has yet been described that inhibits this assay, so it has no negative control. A completely different interpretation is possible rejecting the notion that LAMs traffic sterol across the contact site at all: it could be that $L A M$ genes affect the relative size of sterol pools in the plasma membrane ${ }^{[42]}$, for example by effects on saturated plasma membrane lipids such as sphingolipids ${ }^{[43]}$. These changes would then alter the proportion of sterol trafficked but not the absolute amount ${ }^{[39]}$. Thus, it cannot be said whether the role of LAMs in sterol traffic between ER and plasma membrane is direct or indirect.

Similar to Lam2p, Lam6p might plausibly mediate sterol traffic to/from mitochondria, vacuole or both, since the inner mitochondrial matrix and the vacuolar membrane need sterol, having approximately $40 \%$ and $60 \%$ the level of sterol as the ER respectively ${ }^{[44]}$. Vacuolar sterol is required for vacuole-vacuole fusion ${ }^{[45]}$. Sterol is also needed for the formation of sterol-rich large (micron scale) inwardly curved 
vacuolar domains appearing in cells starved for glucose or stressed, for example by weak acids ${ }^{[46]}$. The formation of these domains requires Lam6p when cells are glucose starved, not under weak acid stress, indicating that Lam6p acts on a subset of multiple pathways underlying their formation ${ }^{[18]}$. Interestingly, these domains spontaneously appear in cells even when growing in log-phase with glucose and without stress solely by forcing Lam $6 p$ to the NVJ and VancE contacts either by removing its mitochondrial binding partners or by deleting the PH-like domain ${ }^{[18]}$. This functional link between Lam6p and the sterol-rich domains might be explained by a role in bulk sterol transport (Fig. 1C). However, as for LAMs at the plasma membrane, an alternative is that Lam6p acts as a "dynamic organizer" of the membrane ${ }^{[18]}$, involving sensing not traffic of sterol (Fig. 1B).

\section{Precise positioning of StARkin domains}

LAMs are located at contact sites that are all $\geq 15 \mathrm{~nm}$ in width (Fig. 3$)^{[47-49]}$. One question that arises is whether they can reach out far enough to act at the far side of such gaps. This was tested for Lam $2 p$, the lack of which causes yeast to be hypersensitive to Amphotericin $B$, an ergosterol sequestering antifungal molecule (the same phenotype is found with loss of Lam1p or Lam3p, but not for Lam4-6p). The deletion phenotype of $\Delta / a m 1 / 2 / 3$ is rescued by expression of soluble StARkin domains from Lam2/4/6p and hLAMa. As seen with other lipid transfer proteins ${ }^{[50]}$, rescue by soluble non-targeted protein requires high level expression compared to the membrane targeted native protein. Significantly, there was also marginal rescue by MLN64 (a sterol specific human StART), but not by StARTs specific for other lipids ${ }^{[20]}$. This suggests that Lam2p functions not just by protein mediated signalling, but this is only one experiment and more must be done to confirm what Lam $2 p$ is doing.

One point that arises about the membrane targeting of LAMs is that the linkers between the StARkin domain and the transmembrane helix are all quite short (Fig. 2). This is particularly true for Lam2p, where the linker is 59 residues long, of which 18 might form a polybasic domain that binds to the plasma membrane, leaving only 41 residues, which can extend to a maximum of $16 \mathrm{~nm}$ (3.8 A per residue ${ }^{[51]}$, see insert in Fig. 2). Linking the second StARkin domain of Lam2p to the ER with a series of linkers of different lengths ( 6 to 103 aa, equivalent to 2 to $39 \mathrm{~nm}$ ) of flexible, unstructured polypeptide showed that linkers much shorter than the natural one inhibit the activity, while a linker that maximally stretches $15 \mathrm{~nm}$ was partly active, and linkers stretching up to $27 \mathrm{~nm}$ or more produce maximal activity ${ }^{[20]}$. This indicates that the StARkin domain must reach out to the plasma membrane to function. Relative distance from the anchor is not everything, as shown by considering the first StARkin domain of Lam2p. The first domain is almost as active as the second domain when expressed on its own, but the second domain alone is necessary and sufficient for rescue of function: deletion of the first StARkin domain has no phenotypic effect, but deletion (or point mutation) of the second StARkin domain kills activity of the whole protein ${ }^{[20]}$. This indicates that the context of StARkin domains within full-length LAM proteins is crucial for function.

\section{Summary:}

Just three papers have been published on the cell biology of the LAM family ${ }^{[18,20,27]}$. The emphasis here is therefore on new-ness: finding the new proteins has lead to new understandings of intracellular architecture and even new consideration of a whole protein superfamily. However, there is still the same old conundrum about what proteins that can solubilise membrane lipids actually do with them. Both the Amphotericin B sensitivity accompanying $L A M 1 / 2 / 3$ deletion and the vacuolar changes accompanying deletion of $L A M 6$ could come from altered sterol traffic or from altered regulation of its distribution between different pools ${ }^{[18,20]}$. Resolving this question may require the development of new technologies that allow us to follow the extremely fast intracellular traffic of lipids.

\section{ACKNOWLEDGEMENTS:}

We thank Alberto Gatta and Matt Hayes for sharing unpublished data. 


\section{FUNDING:}

LHW is funded by an iCASE award from the MRC (MR/J006580/1). 


\section{Box 1: Definition of a lipid transfer protein}

It is important to understand that the term "lipid transfer protein" was originally defined as an in vitro biochemical activity that mediates quasi enzymatic transfer of lipid from donor to acceptor lipid bilayers

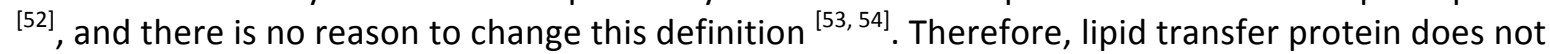
describe in vivo function: just because it can transfer lipid, it does not follow that it does transfer lipid. It might only bind lipid, acting as a sensor that converts lipid concentration into a conformational change $[55,56]$. In fact, since all lipid binding is likely to be accompanied by some conformational change in the protein, any lipid transfer protein may convey a signal, either intra- or intermolecular, that indicates a sensor function. And so the question facing cell biologists is whether experimental evidence can be obtained to show if any lipid transfer protein transfers lipid in vivo.

\section{Box 2. StARkin: a new start for naming the kin of StAR}

Six families were identified as a conserved protein domain superfamily by NCBI as helix grip folds, all consisting of a curved 7-stranded beta sheet with one C-terminal alpha helix and a broken helix between $\beta 1$ and $\beta 2$ that is not in contact with the sheet and makes a large internal cavity. Proteins across families share so little sequence that the homology cannot be detected from conventional sequence alignment tools such as PSI-BLAST ${ }^{[57]}$. Instead, the superfamily was built by aligning 3D structures, and DALI shows RMSD $\leq 4$ Angstroms throughout. Alignment by HHblits, which uses Hidden Markov Models and is more sensitive than PSI-BLAST ${ }^{[58]}$, shows that several of these families can be linked by sequence alone (Table 2A).

The superfamily has been named either StART after the single best known family ${ }^{[34]}$, or Bet v1-like ${ }^{[16]}$, or SRPBCC - an acronym for six founder family members introduced via the NCBI Conserved Domain Database (StART/RHOalphaC/PITP/Bet v1/CoxG/CalC) ${ }^{[59]}$. As described by Aravind and co-workers, the $\mathrm{RHO \alpha C} /$ naphthalene dioxygenase family should not be included with the others, as it does not come up as a structural homologue of any other member (Table 2). New branches of the established families can be added for proteins families with recognizably related sequence: PYR/PYL/RCAR abscissic acid receptors in plants ${ }^{[60]}$, and DUF3211 fatty acid binding proteins in archaea ${ }^{[61]}$. More significantly, three whole new families have been added: (i) LAMs ${ }^{[18,20,27]}$. In the context of a study in Arabidopsis, where one gene is called VAD-1 (Fig. 1) because its deletion causes vascular associated death of leaves, the lipid transfer domains have been called VASt domains for VAD1 Analog of StAR-related lipid transfer ${ }^{[16]}$ However, because this name is inextricably linked to plants we do not think it is a helpful way to describe the whole family. (ii) PRELI domains, which bind and transfer phospholipids such as phosphatidic acid, found both as domains in cytoplasmic proteins and as single domain proteins in the inter-membrane space of mitochondria where they are stabilised a second, small protein (TRIAP/Mdm35) ${ }^{[62]}$ to make an overall structure similar to PITP ${ }^{[63-65]}$; (iii) DUF3809 proteins in archaea (Joint Center for Structural Genomics, structure released 2010). These families are not identified by alignments of sequence alone (Table $2 \mathrm{~A}$ ), but are detected by structural bioinformatic tools such as HHsearch (Table 2B/C).

The problem with RHO $\alpha$ C suggests that the (albeit little used) SRPBCC acronym should be dropped. The other widely used name, StART superfamily ${ }^{[34]}$, is reasonable on scientific grounds, but it overvalues StARTs compared to other families, and the ubiquity of the word "start" in internet search tools makes it hard for entrants to the field to find the right resources quickly. We therefore invite researchers in the field to suggest new names for this growing superfamily. Our own suggestion is StARkin, the concept of the superfamily being contained in the suffix kin, which means relatives or family. 
Table 1: Fifteen folds of intracellular proteins that bind lipids

\author{
all alpha $\quad \operatorname{Coq} 9$ 9, GLTP $^{\ddagger}$, saposin*

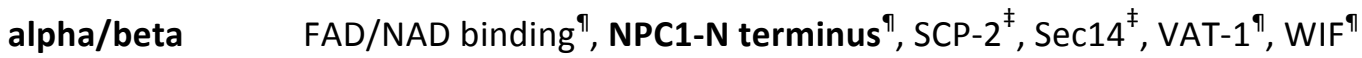 \\ mainly beta $\quad$ OSBP-related ${ }^{\ddagger}$, SRPBCC [StART, PITP, Bet v1, LAM etc. $]^{\ddagger}$, TULIP (SMP) ${ }^{\natural}$ \\ almost all beta lipocalin/calycin* \\ all beta $\quad$ FABP*, NPC2[GDI]*
}

Proteins that fold in such a way as to shield and solubilise hydrophobic ligands in the cytoplasm are identified by the predominant secondary structural element in their fold. Five of these families were included in a previous review ${ }^{\ddagger}[66]$, others were omitted because the ligands are only mono-acyl lipids* ${ }^{[67-70]}$, or because they were discovered since ${ }^{914,15,71-74]}$. The high rate of new discovery of proteins that can solubilize lipids suggests that many more are yet to be discovered. Alternate names include:

CRAL/TRIO for Sec14, nonspecific lipid transfer protein (nsLTP) for SCP-2 (sterol carrier protein 2), and StARkin for the family including StART, PITP Bet v1, LAM etc. sometimes called SRPBCC (see Box 2). 
Table 2: Sequence and structural similarities in the StARkin superfamily

A Hblits (E-values)
\begin{tabular}{|l|c|c|c|c|c|c|c|c|c|c|c|}
\hline Q T & StART & RHO & PITP & BetvI & CoxG & CaIC & RCAR & D 3211 & PRELI & LAM & D 3809 \\
\hline StART & -43 & & & -3 & -6 & 1 & -4 & 3 & 3 & 2 & \\
\hline RHO & & -38 & & & & & & & & & \\
\hline PITP & & & -126 & & & & & & 0 & & \\
\hline Betvl & -3 & & & -30 & -15 & -14 & -19 & 0 & & 2 & 4 \\
\hline CoxG & -6 & & & -15 & -32 & -11 & -16 & -6 & 3 & 3 & 2 \\
\hline CalC & -2 & & & -14 & -15 & -17 & -16 & -3 & & 1 & \\
\hline RCAR & -5 & & & -19 & -16 & -13 & -31 & 0 & 4 & 2 & \\
\hline DUF3211 & -2 & & & -5 & -20 & -10 & -8 & -14 & & 2 & 2 \\
\hline PRELI & 2 & & -1 & 1 & 1 & 3 & 3 & 3 & -66 & 2 & \\
\hline LAM & 1 & & & 0 & 1 & 2 & 2 & & 2 & -35 & \\
\hline DUF3809 & & & & & 0 & & 3 & 3 & & & -76 \\
\hline
\end{tabular}

B HHsearch (probability of shared structure)

\begin{tabular}{|l|l|l|l|l|c|c|c|c|c|c|c|}
\multicolumn{1}{c|}{ QT } & StART & RHO & PITP & Betvl & CoxG & CaIC & RCAR & D3211 & PRELI & LAM & D3809 \\
\hline StART & 100 & & & 98 & 99 & 44 & 98 & 2 & 18 & 17 & \\
\hline RHO & & 100 & & & & & & & & & \\
\hline PITP & & & 100 & & & & & & 94 & & \\
\hline Betvl & 98 & & & 100 & 100 & 100 & 100 & 96 & & 10 & 1 \\
\hline CoxG & 99 & & & 100 & 100 & 99 & 100 & 99 & 1 & 3 & 32 \\
\hline CalC & 97 & & & 100 & 100 & 100 & 100 & 98 & & 21 & \\
\hline RCAR & 99 & & & 100 & 100 & 100 & 100 & 95 & 1 & 10 & \\
\hline DUF3211 & 98 & & & 99 & 100 & 99 & 99 & 100 & & 8 & 53 \\
\hline PRELI & 39 & & 97 & 89 & 77 & 1 & 9 & 4 & 100 & 17 & \\
\hline LAM & 76 & & & 93 & 88 & 5 & 42 & & 45 & 100 & \\
\hline DUF3809 & & & & & 95 & & 5 & 5 & & & 100 \\
\hline
\end{tabular}

CH Hearch (number of columns matched)
\begin{tabular}{|l|c|c|c|c|c|c|c|c|c|c|c|}
\hline Q & StART & RHO & PITP & Betvl & CoxG & CaIC & RCAR & D3211 & PRELI & LAM & D 3809 \\
\hline StART & 208 & & & 142 & 137 & 140 & 144 & 124 & 149 & 130 & \\
\hline RHO & & 192 & & & & & & & & & \\
\hline PITP & & & 270 & & & & & & 127 & & \\
\hline Betvl & 145 & & & 157 & 139 & 142 & 147 & 102 & & 115 & 40 \\
\hline CoxG & 138 & & & 141 & 157 & 134 & 137 & 124 & 114 & 110 & 135 \\
\hline CalC & 142 & & & 144 & 136 & 150 & 142 & 122 & & 109 & \\
\hline RCAR & 147 & & & 146 & 138 & 139 & 183 & 122 & 120 & 108 & \\
\hline DUF3211 & 128 & & & 99 & 130 & 97 & 129 & 137 & & 33 & 125 \\
\hline PRELI & 150 & & 126 & 141 & 112 & 23 & 138 & 36 & 174 & 132 & \\
\hline LAM & 88 & & & 148 & 142 & 149 & 147 & & 162 & 150 & \\
\hline DUF3809 & & & & & 137 & & 134 & 117 & & & 158 \\
\hline
\end{tabular}

Proteins with solved structures (except for LAMs) that have been assigned to different StARkin families were aligned with each other at toolkit.tuebingen.mpg.de/ (A) HHblits ${ }^{[58]}$ was used (8 iterations) for multiple sequence alignment (numbers show $\log _{10}$ of e-value of the significance of the match, colour spectrum red $\leq-20 \Leftrightarrow$ yellow $=-6 \Leftrightarrow$ white $\geq 0$ ). (B) and (C) HHsearch ${ }^{[19]}$ seeded with these alignments produced both (B) the probability of shared structure (colour spectrum red $=100 \Leftrightarrow$ yellow $=80 \Leftrightarrow$ white $\leq 10$; highly reliable $\geq 80 \%$ ); and (C) the number of columns matched (colour spectrum red $\geq 170 \Leftrightarrow$ yellow $=125 \Leftrightarrow$ white $\leq 85$ ). Sequences used: six SRPBCCs (StART: 1em2, RHO $\alpha \mathrm{C}$ (dioxygenase): $2 \mathrm{bmo}$, PITP: 1kcm, Bet v1: 4bk7, CoxG: 2ns9, CalC: 1zxf), two related helix grips (PYR/PYL/RCAR: 3rt2, and DUF3211: 2ejx), and three proteins detected by structural bioinformatics (PRELI: 4ytw_b, LAM: Lam1p $\neq$, and DUF3809: 3nqn, an archaeal protein of unknown function). Query sequences (Q) are in the first column, and targets (T) are shown across the other columns. * The target LAM sequence is not a solved structure, hence we used an entry in the PFAM database that includes the domain designated as DUF4782 (PF16016) in its most recent release (v28.0, May 2015). Note that RHO $\alpha \mathrm{C}$ is unrelated to all others, even in HHsearch, and should not be included in this superfamily, as suggested previously ${ }^{[34]}$. RCAR and DUF3211 are related by sequence, i.e. in (A), to CoxG, so they should not be considered as separate families. Applying that rule using a HHblits threshold $\leq 10^{-10}$ to identify sequence homology places all of Bet v1, CoxG, CalC, RCAR and DUF3211 in one single family of sequences. On this basis the minimal StARkin superfamily has six members: StART, PITP, Bet v1, PRELI, LAM, and DUF3809. 


\section{Legends:}

Fig. 1: The function of membrane anchored lipid transfer proteins

A. A soluble lipid transfer protein (grey blue)formed of a box with a lid and a mainly hydrophobic cavity that picks up and drops off a specific lipids (here a sterol) ${ }^{[1]}$ or possibly binds (exchanges, not shown) two lipids ${ }^{[40,53]}$. Diffusion through the cytoplasm (black arrows) limits the rate of limit transfer, as it takes $160 \mathrm{~ms}$ for a small protein to cross 1 micron. Pick up and drop off reactions involving reversible opening of the structure (white arrows, duration unknown "?") ${ }^{[38]}$ are not strictly symmetrical, as internal lipid alters protein structure*, particularly the lid, and lid opening can depend on both lipid bound and membrane composition ${ }^{[40]}$. B. Membrane anchoring (red) changes lipid transfer protein activity (green), so that in the absence of an adjacent second organelle it can only reach out a limited distance from the inserted membrane. Possible functions include sensing lipid to convey either unbound or bound* state to a downstream target (orange) ${ }^{[54]}$, or inducing intermediate states where lipids half in and half out of the lipid transfer protein are presented to a protein that cannot probe the bilayer (light grey) ${ }^{[53]}$. C. At a membrane contact site, a lipid transfer protein might contain domains that form a bridge (red, note this arrangement of bridging and transfer domains is only theoretical) that crosses contacts 10-30 nm wide (not drawn to scale). Speed of transfer by an anchored lipid transfer domain (green) is limited by pick up and drop off, not by diffusion ( $\leq 0.2 \mathrm{~ms})$. In yeast plasma membrane there is segregation of sterol and saturated lipids (grey) away from unsaturated lipids (pink) ${ }^{[75]}$, which may be mediated by lipid shells around transmembrane helices of plasma membrane proteins (not shown) ${ }^{[76]}$, so that concentration of free sterol is no higher than the $E R^{[37]}$. Effects on this segregation alter the relative and absolute sizes of sterol pools(horizontal arrows) ${ }^{[42]}$, changing the concentration of plasma membrane sterol accessible to lipid transfer proteins ${ }^{[77]}$.

\section{Fig. 2: Large families of LAMs in different eukaryotes}

Humans, budding yeast and thale cress have 3, 6 and 4 LAM proteins with domain composition as shown in the key. A functionally key aspect is the presence of a transmembrane helix. Usually this is at the C-terminus near the StARkin domain, but in some plant proteins the pattern is reversed. The transmembrane helix in Lam4p* is only weakly predicted by tools such as TOPCONS, but the protein shows the similar targeting to portions of the ER as Lam1-3p. Typically there is also a PH-like domain from the GRAM family upstream of the StARkin lipid transfer domain ${ }^{[16]}$. Insert shows an enlargement of the linker between the second StARkin domain and TMH of Lam2p, with the number of amino acids in both the poly-basic region and the remaining linker. For all LAM domains the number of charges in the immediately adjacent linker is shown: overall positive in blue, overall negative in red and close to neutral in grey. The helix preceding the $\mathrm{PH}$-like domain has a consensus as shown in the key, where upper case: predominant residue, lower case: propensity; $+/-/ \pm=$ charged as indicated; $\partial=$ hydrophilic uncharged; $\varnothing=$ hydrophobic; $x=$ any.

\section{Fig. 3: LAMs target four different membrane contact sites in yeast}

Diagram of Lam targeting to different contacts, not drawn to scale. Lam5/6p (red) localises to three different intracellular membrane contact sites whilst retained on the ER membrane via a transmembrane helix. Lam6p localisation to NVJ and VancE contacts occurs via interaction with Vac8p. Lam6 $p$ also localises to ER-mitochondria contacts where its PH-like domain interacts with Tom70/71p. Lam $5 p$ has the same targeting as Lam6p when over-expressed (inset, GFP-Lam5p co-expressed with RFP-Tom6p on the outer mitochondrial membrane and vacuolar lumen, likely as a result of mitophagy), though its distribution when expressed at endogenous levels is not known. Thus far, Lam1-4p is only known to localise at one contact site formed by the ER and the plasma membrane. Lam2/4p (blue) consists of two StARkin domains and one PH-like domain which are all able to reach in trans to the plasma membrane (PM) whilst tethered by a single transmembrane helix to the ER. Lam3p (yellow) possesses two transmembrane helix with very little ER luminal domain. Lam3p has a single StART domain, two PH-like domains and a BAR domain which are all spatially able to interact with the plasma 
membrane. Inset shows a cell expressing GFP-Lam2p at punctate ER-plasma membrane contacts and coexpressing RFP-ER. Both GFP images are also shown as black on white background. Confocal microscopy images courtesy of Alberto Gatta.

\section{REFERENCES}

1. Wirtz, K.W. and Zilversmit, D.B. (1968) Exchange of phospholipids between liver mitochondria and microsomes in vitro. J Biol Chem. 243, 3596-602

2. Pagano, R.E. (1990) Lipid traffic in eukaryotic cells: mechanisms for intracellular transport and organelle-specific enrichment of lipids. Curr Opin Cell Biol. 2, 652-63

3. Vance, J.E. (1990) Phospholipid synthesis in a membrane fraction associated with mitochondria. J Biol Chem. 265, 7248-56

4. Bernhard, W. and Rouiller, C. (1956) Close topographical relationship between mitochondria and ergastoplasm of liver cells in a definite phase of cellular activity. J Biophys Biochem Cytol. 2, 73-8

5. Prinz, W.A. (2014) Bridging the gap: membrane contact sites in signaling, metabolism, and organelle dynamics. J Cell Biol. 205, 759-69

6. Vihtelic, T.S., Goebl, M., Milligan, S., O'Tousa, J.E., and Hyde, D.R. (1993) Localization of Drosophila retinal degeneration $B$, a membrane-associated phosphatidylinositol transfer protein. J Cell Biol. 122, 1013-22

7. Levine, T.P. and Munro, S. (2001) Dual targeting of Osh1p, a yeast homologue of oxysterolbinding protein, to both the Golgi and the nucleus-vacuole junction. Mol Biol Cell. 12, 1633-44

8. Loewen, C.J., Roy, A., and Levine, T.P. (2003) A conserved ER targeting motif in three families of lipid binding proteins and in Opi1p binds VAP. EMBO J. 22, 2025-35

9. Olkkonen, V.M. and Levine, T.P. (2004) Oxysterol binding proteins: in more than one place at one time? Biochem Cell Biol. 82, 87-98

10. Sullivan, D.P., Ohvo-Rekilä, H., Baumann, N.A., Beh, C.T., and Menon, A.K. (2006) Sterol trafficking between the endoplasmic reticulum and plasma membrane in yeast. Biochem Soc Trans. 34, 358-8

11. Giordano, F., Saheki, Y., Idevall-Hagren, O., Colombo, S.F., Pirruccello, M., Milosevic, I., Gracheva, E.O., Bagriantsev, S.N., Borgese, N., and De Camilli, P. (2013) PI(4,5)P(2)-dependent and $\mathrm{Ca}(2+)$-regulated ER-PM interactions mediated by the extended synaptotagmins. Cell. 153, 1494-509

12. Schrick, K., Nguyen, D., Karlowski, W.M., and Mayer, K.F. (2004) START lipid/sterol-binding domains are amplified in plants and are predominantly associated with homeodomain transcription factors. Genome Biol. 5, R41

13. Lee, I. and Hong, W. (2006) Diverse membrane-associated proteins contain a novel SMP domain. Faseb J. 20, 202-6

14. Kopec, K.O., Alva, V., and Lupas, A.N. (2010) Homology of SMP domains to the TULIP superfamily of lipid-binding proteins provides a structural basis for lipid exchange between ER and mitochondria. Bioinformatics. 26, 1927-31

15. Schauder, C.M., Wu, X., Saheki, Y., Narayanaswamy, P., Torta, F., Wenk, M.R., De Camilli, P., and Reinisch, K.M. (2014) Structure of a lipid-bound extended synaptotagmin indicates a role in lipid transfer. Nature. 510, 552-5

16. Khafif, M., Cottret, L., Balague, C., and Raffaele, S. (2014) Identification and phylogenetic analyses of VASt, an uncharacterized protein domain associated with lipid-binding domains in Eukaryotes. BMC Bioinformatics. 15, 222

17. Roy, A., Kucukural, A., and Zhang, Y. (2010) I-TASSER: a unified platform for automated protein structure and function prediction. Nat Protoc. 5, 725-38

18. Murley, A., Sarsam, R.D., Toulmay, A., Yamada, J., Prinz, W.A., and Nunnari, J. (2015) Ltc1 is an ER-localized sterol transporter and a component of ER-mitochondria and ER-vacuole contacts. J Cell Biol. 209, 539-48

19. Soding, J., Biegert, A., and Lupas, A.N. (2005) The HHpred interactive server for protein homology detection and structure prediction. Nucleic Acids Res. 33, W244-8 
20. Gatta, A.T., Wong, L.H., Sere, Y.Y., Calderon-Norena, D.M., Cockcroft, S., Menon, A.K., and Levine, T.P. (2015) A new family of StART domain proteins at membrane contact sites has a role in ER-PM sterol transport. Elife. 4, e07253

21. Kellis, M., Birren, B.W., and Lander, E.S. (2004) Proof and evolutionary analysis of ancient genome duplication in the yeast Saccharomyces cerevisiae. Nature. 428, 617-24

22. Di Bernardo, M.C., Crowther-Swanepoel, D., Broderick, P., Webb, E., Sellick, G., Wild, R., Sullivan, K., Vijayakrishnan, J., Wang, Y., Pittman, A.M., et al. (2008) A genome-wide association study identifies six susceptibility loci for chronic lymphocytic leukemia. Nat Genet. 40, 1204-10

23. Wu, S.Y., Yang, X., Gharpure, K.M., Hatakeyama, H., Egli, M., McGuire, M.H., Nagaraja, A.S., Miyake, T.M., Rupaimoole, R., Pecot, C.V., et al. (2014) 2'-OMe-phosphorodithioate-modified siRNAs show increased loading into the RISC complex and enhanced anti-tumour activity. Nat Commun. 5, 3459

24. Yu, J.W., Mendrola, J.M., Audhya, A., Singh, S., Keleti, D., DeWald, D.B., Murray, D., Emr, S.D., and Lemmon, M.A. (2004) Genome-wide analysis of membrane targeting by S. cerevisiae pleckstrin homology domains. Mol Cell. 13, 677-88

25. Schrick, K., Bruno, M., Khosla, A., Cox, P.N., Marlatt, S.A., Roque, R.A., Nguyen, H.C., He, C., Snyder, M.P., Singh, D., et al. (2014) Shared functions of plant and mammalian StAR-related lipid transfer (START) domains in modulating transcription factor activity. BMC Biol. 12, 70

26. de Saint-Jean, M., Delfosse, V., Douguet, D., Chicanne, G., Payrastre, B., Bourguet, W., Antonny, B., and Drin, G. (2011) Osh4p exchanges sterols for phosphatidylinositol 4-phosphate between lipid bilayers. J Cell Biol. 195, 965-78

27. Elbaz-Alon, Y., Eisenberg-Bord, M., Shinder, V., Stiller, S.B., Shimoni, E., Wiedemann, N., Geiger, T., and Schuldiner, M. (2015) Lam6 Regulates the Extent of Contacts between Organelles. Cell Rep. 12, 7-14

28. Wu, C.H., Lee, S.C., and Wang, C.W. (2011) Viral protein targeting to the cortical endoplasmic reticulum is required for cell-cell spreading in plants. J Cell Biol. 193, 521-35

29. Manford, A.G., Stefan, C.J., Yuan, H.L., Macgurn, J.A., and Emr, S.D. (2012) ER-to-plasma membrane tethering proteins regulate cell signaling and ER morphology. Dev Cell. 23, 1129-40

30. Pan, X., Roberts, P., Chen, Y., Kvam, E., Shulga, N., Huang, K., Lemmon, S., and Goldfarb, D.S. (2000) Nucleus-vacuole junctions in Saccharomyces cerevisiae are formed through the direct interaction of Vac8p with Nvj1p. Mol Biol Cell. 11, 2445-57

31. Elbaz-Alon, Y., Rosenfeld-Gur, E., Shinder, V., Futerman, A.H., Geiger, T., and Schuldiner, M. (2014) A dynamic interface between vacuoles and mitochondria in yeast. Dev Cell. 30, 95-102

32. Honscher, C., Mari, M., Auffarth, K., Bohnert, M., Griffith, J., Geerts, W., van der Laan, M., Cabrera, M., Reggiori, F., and Ungermann, C. (2014) Cellular metabolism regulates contact sites between vacuoles and mitochondria. Dev Cell. 30, 86-94

33. Henne, W.M., Zhu, L., Balogi, Z., Stefan, C., Pleiss, J.A., and Emr, S.D. (2015) Mdm1/Snx13 is a novel ER-endolysosomal interorganelle tethering protein. J Cell Biol. 210, 541-51

34. Iyer, L.M., Koonin, E.V., and Aravind, L. (2001) Adaptations of the helix-grip fold for ligand binding and catalysis in the START domain superfamily. Proteins. 43, 134-44

35. Tang, F., Peng, Y., Nau, J.J., Kauffman, E.J., and Weisman, L.S. (2006) Vac8p, an armadillo repeat protein, coordinates vacuole inheritance with multiple vacuolar processes. Traffic. 7, 1368-77

36. Suresh, H.G., da Silveira Dos Santos, A.X., Kukulski, W., Tyedmers, J., Riezman, H., Bukau, B., and Mogk, A. (2015) Prolonged starvation drives reversible sequestration of lipid biosynthetic enzymes and organelle reorganization in Saccharomyces cerevisiae. Mol Biol Cell. 26, 1601-15

37. Lange, Y., Ye, J., Rigney, M., and Steck, T.L. (1999) Regulation of endoplasmic reticulum cholesterol by plasma membrane cholesterol. J Lipid Res. 40, 2264-70

38. Iaea, D.B., Dikiy, I., Kiburu, I., Eliezer, D., and Maxfield, F.R. (2015) STARD4 Membrane Interactions and Sterol Binding. Biochemistry. 54, 4623-36

39. Georgiev, A., Sullivan, D.P., Kersting, M.C., Dittman, J.S., Beh, C.T., and Menon, A.K. (2011) Osh proteins regulate membrane sterol organization but are not required for sterol movement between the ER and PM. Traffic. 12, 1341-1355 
40. Moser von Filseck, J., Vanni, S., Mesmin, B., Antonny, B., and Drin, G. (2015) A phosphatidylinositol-4-phosphate powered exchange mechanism to create a lipid gradient between membranes. Nat Commun. 6, 6671

41. Li, Y. and Prinz, W.A. (2004) ATP-binding cassette (ABC) transporters mediate nonvesicular, raftmodulated sterol movement from the plasma membrane to the endoplasmic reticulum. J Biol Chem. 279, 45226-34

42. Das, A., Brown, M.S., Anderson, D.D., Goldstein, J.L., and Radhakrishnan, A. (2014) Three pools of plasma membrane cholesterol and their relation to cholesterol homeostasis. Elife. 3, e02882

43. Vecer, J., Vesela, P., Malinsky, J., and Herman, P. (2014) Sphingolipid levels crucially modulate lateral microdomain organization of plasma membrane in living yeast. FEBS Lett. 588, 443-9

44. Schneiter, R., Brugger, B., Sandhoff, R., Zellnig, G., Leber, A., Lampl, M., Athenstaedt, K., Hrastnik, C., Eder, S., Daum, G., et al. (1999) Electrospray ionization tandem mass spectrometry (ESI-MS/MS) analysis of the lipid molecular species composition of yeast subcellular membranes reveals acyl chain-based sorting/remodeling of distinct molecular species en route to the plasma membrane. J Cell Biol. 146, 741-54

45. Zick, M., Stroupe, C., Orr, A., Douville, D., and Wickner, W.T. (2014) Membranes linked by transSNARE complexes require lipids prone to non-bilayer structure for progression to fusion. Elife. 3, e01879

46. Toulmay, A. and Prinz, W.A. (2013) Direct imaging reveals stable, micrometer-scale lipid domains that segregate proteins in live cells. J Cell Biol. 202, 35-44

47. West, M., Zurek, N., Hoenger, A., and Voeltz, G.K. (2011) A 3D analysis of yeast ER structure reveals how ER domains are organized by membrane curvature. J Cell Biol. 193, 333-46

48. Friedman, J.R., Lackner, L.L., West, M., DiBenedetto, J.R., Nunnari, J., and Voeltz, G.K. (2011) ER tubules mark sites of mitochondrial division. Science. 334, 358-62

49. Millen, J.I., Pierson, J., Kvam, E., Olsen, L.J., and Goldfarb, D.S. (2008) The luminal N-terminus of yeast Nvj1 is an inner nuclear membrane anchor. Traffic. 9, 1653-64

50. Hanada, K., Kumagai, K., Yasuda, S., Miura, Y., Kawano, M., Fukasawa, M., and Nishijima, M. (2003) Molecular machinery for non-vesicular trafficking of ceramide. Nature. 426, 803-9

51. Pillardy, J., Czaplewski, C., Liwo, A., Lee, J., Ripoll, D.R., Kazmierkiewicz, R., Oldziej, S., Wedemeyer, W.J., Gibson, K.D., Arnautova, Y.A., et al. (2001) Recent improvements in prediction of protein structure by global optimization of a potential energy function. Proc Natl Acad Sci U S A. 98, 2329-33

52. Wirtz, K.W. (1991) Phospholipid transfer proteins. Annu Rev Biochem. 60, 73-99

53. Grabon, A., Khan, D., and Bankaitis, V.A. (2015) Phosphatidylinositol transfer proteins and instructive regulation of lipid kinase biology. Biochim Biophys Acta. 1851, 724-35

54. Mousley, C.J., Yuan, P., Gaur, N.A., Trettin, K.D., Nile, A.H., Deminoff, S.J., Dewar, B.J., Wolpert, M., Macdonald, J.M., Herman, P.K., et al. (2012) A sterol-binding protein integrates endosomal lipid metabolism with TOR signaling and nitrogen sensing. Cell. 148, 702-15

55. Wang, P.Y., Weng, J., and Anderson, R.G. (2005) OSBP is a cholesterol-regulated scaffolding protein in control of ERK 1/2 activation. Science. 307, 1472-6

56. Bankaitis, V.A., Mousley, C.J., and Schaaf, G. (2010) The Sec14 superfamily and mechanisms for crosstalk between lipid metabolism and lipid signaling. Trends Biochem Sci. 35, 150-60

57. Radauer, C., Lackner, P., and Breiteneder, H. (2008) The Bet v 1 fold: an ancient, versatile scaffold for binding of large, hydrophobic ligands. BMC Evol Biol. 8, 286

58. Remmert, M., Biegert, A., Hauser, A., and Soding, J. (2012) HHblits: lightning-fast iterative protein sequence searching by HMM-HMM alignment. Nat Methods. 9, 173-5

59. Marchler-Bauer, A., Anderson, J.B., Cherukuri, P.F., DeWeese-Scott, C., Geer, L.Y., Gwadz, M., He, S., Hurwitz, D.I., Jackson, J.D., Ke, Z., et al. (2005) CDD: a Conserved Domain Database for protein classification. Nucleic Acids Res. 33, D192-6

60. Ma, Y., Szostkiewicz, I., Korte, A., Moes, D., Yang, Y., Christmann, A., and Grill, E. (2009) Regulators of PP2C phosphatase activity function as abscisic acid sensors. Science. 324, 1064-8 
61. Miyakawa, T., Sawano, Y., Miyazono, K., Miyauchi, Y., Hatano, K., and Tanokura, M. (2013) A thermoacidophile-specific protein family, DUF3211, functions as a fatty acid carrier with novel binding mode. J Bacteriol. 195, 4005-12

62. Connerth, M., Tatsuta, T., Haag, M., Klecker, T., Westermann, B., and Langer, T. (2012) Intramitochondrial transport of phosphatidic acid in yeast by a lipid transfer protein. Science. 338, 815-8

63. Miliara, X., Garnett, J.A., Tatsuta, T., Abid Ali, F., Baldie, H., Perez-Dorado, I., Simpson, P., Yague, E., Langer, T., and Matthews, S. (2015) Structural insight into the TRIAP1/PRELI-like domain family of mitochondrial phospholipid transfer complexes. EMBO Rep. 16, 824-35

64. Watanabe, Y., Tamura, Y., Kawano, S., and Endo, T. (2015) Structural and mechanistic insights into phospholipid transfer by Ups1-Mdm35 in mitochondria. Nat Commun. 6, 7922

65. Yu, F., He, F., Yao, H., Wang, C., Wang, J., Li, J., Qi, X., Xue, H., Ding, J., and Zhang, P. (2015) Structural basis of intramitochondrial phosphatidic acid transport mediated by Ups1-Mdm35 complex. EMBO Rep. 16, 813-23

66. Holthuis, J.C. and Levine, T.P. (2005) Lipid traffic: floppy drives and a superhighway. Nat Rev Mol Cell Biol. 6, 209-20

67. Ahn, V.E., Faull, K.F., Whitelegge, J.P., Fluharty, A.L., and Prive, G.G. (2003) Crystal structure of saposin B reveals a dimeric shell for lipid binding. Proc Natl Acad Sci U S A. 100, 38-43

68. Hodsdon, M.E., Ponder, J.W., and Cistola, D.P. (1996) The NMR solution structure of intestinal fatty acid-binding protein complexed with palmitate: application of a novel distance geometry algorithm. J Mol Biol. 264, 585-602

69. Breustedt, D.A., Korndorfer, I.P., Redl, B., and Skerra, A. (2005) The 1.8-A crystal structure of human tear lipocalin reveals an extended branched cavity with capacity for multiple ligands. J Biol Chem. 280, 484-93

70. Friedland, N., Liou, H.L., Lobel, P., and Stock, A.M. (2003) Structure of a cholesterol-binding protein deficient in Niemann-Pick type C2 disease. Proc Natl Acad Sci U S A. 100, 2512-7

71. Lohman, D.C., Forouhar, F., Beebe, E.T., Stefely, M.S., Minogue, C.E., Ulbrich, A., Stefely, J.A., Sukumar, S., Luna-Sanchez, M., Jochem, A., et al. (2014) Mitochondrial COQ9 is a lipid-binding protein that associates with COQ7 to enable coenzyme Q biosynthesis. Proc Natl Acad Sci U S A. 111, E4697-705

72. Kachalova, G.S., Bourenkov, G.P., Mengesdorf, T., Schenk, S., Maun, H.R., Burghammer, M., Riekel, C., Decker, K., and Bartunik, H.D. (2010) Crystal structure analysis of free and substratebound 6-hydroxy-L-nicotine oxidase from Arthrobacter nicotinovorans. J Mol Biol. 396, 785-99

73. Junker, M. and Rapoport, T.A. (2015) Involvement of VAT-1 in Phosphatidylserine Transfer from the Endoplasmic Reticulum to Mitochondria. Traffic.

74. Malinauskas, T., Aricescu, A.R., Lu, W., Siebold, C., and Jones, E.Y. (2011) Modular mechanism of Wnt signaling inhibition by Wnt inhibitory factor 1 . Nat Struct Mol Biol. 18, 886-93

75. Malinsky, J., Opekarova, M., and Tanner, W. (2010) The lateral compartmentation of the yeast plasma membrane. Yeast. 27, 473-8

76. Anderson, R.G. and Jacobson, K. (2002) A role for lipid shells in targeting proteins to caveolae, rafts, and other lipid domains. Science. 296, 1821-5

77. Mesmin, B. and Maxfield, F.R. (2009) Intracellular sterol dynamics. Biochim Biophys Acta. 1791, 636-45 